\title{
Principal functions of matrix Sturm-Liouville operators with boundary conditions dependent on the spectral parameter
}

\author{
Deniz Katar, Murat Olgun, and Cafer Coskun
}




\title{
PRINCIPAL FUNCTIONS OF MATRIX STURM-LIOUVILLE OPERATORS WITH BOUNDARY CONDITIONS DEPENDENT ON THE SPECTRAL PARAMETER
}

\author{
DENIZ KATAR, MURAT OLGUN, AND CAFER COSKUN \\ Received 18 March, 2014
}

\begin{abstract}
Let $L$ denote operator generated in $L_{2}\left(\mathbb{R}_{+}, E\right)$ by the differential expression

$$
l(y)=-y^{\prime \prime}+Q(x) y, x \in \mathbb{R}_{+}:=[0, \infty),
$$

and the boundary condition $\left(A_{0}+A_{1} \lambda\right) Y^{\prime}(0, \lambda)-\left(B_{0}+B_{1} \lambda\right) Y(0, \lambda)=0$, where $Q$ is a matrixvalued function and $A_{0}, A_{1}, B_{0}, B_{1}$ are non-singular matrices, with $A_{0} B_{1}-A_{1} B_{0} \neq 0$. In this paper, we investigate the principal functions corresponding to the eigenvalues and the spectral singularities of $L$.
\end{abstract}

2010 Mathematics Subject Classification: 34B24; 34L05; 47A10

Keywords: eigenvalues, spectral singularities, spectral analysis, Sturm-Liouville operator, nonselfadjoint matrix operator, principal functions

\section{INTRODUCTION}

Let us consider the boundary value problem (BVP)

$$
\begin{gathered}
-u^{\prime \prime}+q(x) u=\lambda^{2} u, \quad x \in \mathbb{R}_{+}, \\
u(0)=0,
\end{gathered}
$$

in $L^{2}\left(\mathbb{R}_{+}\right)$, where $\mathrm{q}$ is a complex-valued function. The spectral theory of the BVP (1.1)-(1.2) with continuous and point spectrum was investigated by Naimark [20]. He showed the existence of the spectral singularities in the continuous spectrum of the BVP (1.1)-(1.2). Note that the eigenfunctions and the associated functions (principal functions) corresponding to the spectral singularities are not the elements of $L^{2}\left(\mathbb{R}_{+}\right)$. Also, the spectral singularities belong to the continuous spectrum and are the poles of the resolvent's kernel, but are not the eigenvalues of the BVP (1.1)-(1.2). The spectral singularities in the spectral expansion of the BVP (1.1)-(1.2) in terms of the principal functions have been investigated in [19]. The spectral analysis of the quadratic pencil of Schrödinger, Dirac and Klein-Gordon operators with spectral singularities were studied in [2-18]. The spectral analysis of the non-selfadjoint 
operator, generated in $L^{2}\left(\mathbb{R}_{+}\right)$by (1.1) and the boundary condition

$$
\frac{y^{\prime}(0)}{y(0)}=\frac{\beta_{1} \lambda+\beta_{0}}{\alpha_{1} \lambda+\alpha_{0}}
$$

where $\alpha_{i}, \beta_{i} \in \mathbb{C}, i=0,1$ with $\alpha_{0} \beta_{1}-\alpha_{1} \beta_{0} \neq 0$ were investigated in detail by Bairamov et al. [9]. The all above mentioned papers related with the differential and difference equations are scalar coefficients. Spectral analysis of the self-adjoint differential and difference equations with matrix coefficients are studied in [3,10-13].

Let $E$ be an n-dimensional $(n<\infty)$ Euclidian space with the norm $\|$.$\| and let$ us introduce the Hilbert space $L^{2}\left(\mathbb{R}_{+}, E\right)$ consisting of vector-valued functions with the values in $\mathrm{E}$. We will consider the BVP

$$
\begin{gathered}
-y^{\prime \prime}+Q(x) y=\lambda^{2} y, \quad x \in \mathbb{R}_{+}, \\
y(0)=0,
\end{gathered}
$$

in $L^{2}\left(\mathbb{R}_{+}, E\right)$ where $\mathrm{Q}$ is a non-selfadjoint matrix-valued function (i. e., $\left.Q \neq Q^{*}\right)$. It is clear that, the BVP (1.3)-(1.4) is nonselfadjoint. In [14,21] discrete spectrum of the non-selfadjoint matrix Sturm-Liouville operator and properties of the principal functions corresponding to the eigenvalues and the spectral singularities were investigated.

Let us consider the BVP in $L_{2}\left(\mathbb{R}_{+}, E\right)$

$$
\begin{gathered}
-y^{\prime \prime}+Q(x) y=\lambda^{2} y, \quad x \in \mathbb{R}_{+}, \\
\left(A_{0}+A_{1} \lambda\right) y^{\prime}(0, \lambda)-\left(B_{0}+B_{1} \lambda\right) y(0, \lambda)=0,
\end{gathered}
$$

where $Q$ is a non-singular matrix-valued function and $A_{0}, A_{1}, B_{0}, B_{1}$ are nonselfadjoint matrices such $A_{0} B_{1}-A_{1} B_{0} \neq 0$. In this paper, we aim to investigate the properties of the principal functions corresponding to the eigenvalues and the spectral singularities of the BVP (1.5)-(1.6).

\section{Jost Solution of (1.5)}

We will denote the solution of (1.5) satisfying the condition

$$
\lim _{x \rightarrow \infty} y(x, \lambda) e^{-i \lambda x}=I, \quad \lambda \in \overline{\mathbb{C}}_{+}:=\{\lambda: \lambda \in \mathbb{C}, \operatorname{Im} \lambda \geq 0\}
$$

by $E(x, \lambda)$. The solution $E(x, \lambda)$ is called the Jost solution of (1.5).

Under the condition

$$
\int_{0}^{\infty} x\|Q(x)\| d x<\infty
$$

the Jost solution has a representation

$$
E(x, \lambda)=e^{i \lambda x} I+\int_{x}^{\infty} K(x, t) e^{i \lambda t} d t,
$$


for $\lambda \in \overline{\mathbb{C}}_{+}$, where the kernel matrix function $K(x, t)$ satisfies

$$
\begin{aligned}
K(x, t)= & \frac{1}{2} \int_{\frac{x+t}{2}}^{\infty} Q(s) d s+\frac{1}{2} \int_{x}^{\frac{x+t}{2}} \int_{t+x-s}^{t+s-x} Q(s) K(s, v) d v d s \\
& +\frac{1}{2} \int_{\frac{x+t}{2}}^{\infty} \int_{s}^{t+s-x} Q(s) K(s, v) d v d s .
\end{aligned}
$$

Moreover, $K(x, t)$ is continuously differentiable with respect to its arguments and

$$
\begin{aligned}
\|K(x, t)\| & \leq c \sigma\left(\frac{x+t}{2}\right), \\
\left\|K_{x}(x, t)\right\| & \leq \frac{1}{4}\left\|Q\left(\frac{x+t}{2}\right)\right\|+c \sigma\left(\frac{x+t}{2}\right), \\
\left\|K_{t}(x, t)\right\| & \leq \frac{1}{4}\left\|Q\left(\frac{x+t}{2}\right)\right\|+c \sigma\left(\frac{x+t}{2}\right),
\end{aligned}
$$

where $\sigma(x)=\int_{x}^{\infty}\|Q(s)\| d s$ and $c>0$ is a constant. Therefore, $E(x, \lambda)$ is analytic with respect to $\lambda$ in $\mathbb{C}_{+}:=\{\lambda: \lambda \in \mathbb{C}, \operatorname{Im} \lambda>0\}$ and continuous on the real axis [1].

Let $\hat{E}^{ \pm}(x, \lambda)$ denote the solutions of $(1.5)$ subject to the conditions

$$
\lim _{x \rightarrow \infty} \hat{E}^{ \pm}(x, \lambda) e^{ \pm i \lambda x}=I, \quad \lim _{x \rightarrow \infty} \hat{E}_{x}^{ \pm}(x, \lambda) e^{ \pm i \lambda x}= \pm i \lambda I, \quad \lambda \in \overline{\mathbb{C}}_{ \pm} .
$$

Then

$$
\begin{array}{rlrl}
W\left[E(x, \lambda), \hat{E}^{ \pm}(x, \lambda)\right] & =\mp 2 i \lambda I, & \lambda \in \mathbb{C}_{ \pm}, \\
W[E(x, \lambda), E(x,-\lambda)] & =-2 i \lambda I, \quad \lambda \in \mathbb{R},
\end{array}
$$

where $W\left[f_{1}, f_{2}\right]$ is the Wronskian of $f_{1}$ and $f_{2}$.

Let $\varphi(x, \lambda)$ denote the solution of (1.5) subject to the initial conditions $\varphi(0, \lambda)=$ $A_{0}+A_{1} \lambda, \varphi^{\prime}(0, \lambda)=B_{0}+B_{1} \lambda$. Therefore $\varphi(x, \lambda)$ is an entire function of $\lambda$.

Let us define the following functions:

$$
D_{ \pm}(\lambda)=\varphi(0, \lambda) E_{x}(0, \pm \lambda)-\varphi^{\prime}(0, \lambda) E(0, \pm \lambda) \quad \lambda \in \overline{\mathbb{C}}_{ \pm},
$$

where $\overline{\mathbb{C}}_{ \pm}=\{\lambda: \lambda \in \mathbb{C}, \pm \operatorname{Im} \lambda \geq 0\}$. It is obvious that the functions $D_{+}(\lambda)$ and $D_{-}(\lambda)$ are analytic in $\mathbb{C}_{+}$and $\mathbb{C}_{-}$, respectively, and continuous on the real axis. The functions $D_{+}$and $D_{-}$are called Jost functions of $L$. 


\section{Eigenvalues and Spectral Singularities of $L$}

The resolvent of $L$ defined by

$$
R_{\lambda}(L) f=\int_{0}^{\infty} G(x, t ; \lambda) g(t) d t, \quad g \in L_{2}\left(\mathbb{R}_{+}, E\right),
$$

where

$$
G(x, t ; \lambda)= \begin{cases}G_{+}(x, t ; \lambda), & \lambda \in \mathbb{C}_{+} \\ G_{-}(x, t ; \lambda), & \lambda \in \mathbb{C}_{-},\end{cases}
$$

and

$$
G_{ \pm}(x, t ; \lambda)=\left\{\begin{array}{c}
-E(x, \pm \lambda) D_{ \pm}^{-1}(\lambda) \varphi^{T}(t, \lambda), 0 \leq t \leq x \\
-\varphi(x, \lambda)\left[D_{+}^{T}( \pm \lambda)\right]^{-1} E^{T}(t, \pm \lambda), x \leq t<\infty .
\end{array}\right.
$$

We will show the set of eigenvalues and the set of spectral singularities of the operator $L$ by $\sigma_{d}$ and $\sigma_{s s}$, respectively.

Let us suppose that

$$
H_{ \pm}(\lambda)=\operatorname{det} D_{ \pm}(\lambda)
$$

From (2.3) and (3.1)-(3.4), we get

$$
\begin{aligned}
\sigma_{d} & =\left\{\lambda: \lambda \in \mathbb{C}_{+}, H_{+}(\lambda)=0\right\} \cup\left\{\lambda: \lambda \in \mathbb{C}_{-}, H_{-}(\lambda)=0\right\} \\
\sigma_{s s} & =\left\{\lambda: \lambda \in \mathbb{R}^{*}, H_{+}(\lambda)=0\right\} \cup\left\{\lambda: \lambda \in \mathbb{R}^{*}, H_{-}(\lambda)=0\right\},
\end{aligned}
$$

where $\mathbb{R}^{*}=\mathbb{R} \backslash\{0\}$.

We see from that, the functions

$$
\begin{aligned}
K^{+}(\lambda) & =\frac{\hat{D}_{+}(\lambda)}{2 i \lambda} E(x, \lambda)-\frac{D_{+}(\lambda)}{2 i \lambda} \hat{E}^{+}(x, \lambda), \lambda \in \mathbb{C}_{+}, \\
K^{-}(\lambda) & =\frac{\hat{D}_{-}(\lambda)}{2 i \lambda} E(x,-\lambda)-\frac{D_{-}(\lambda)}{2 i \lambda} \hat{E}^{-}(x, \lambda), \lambda \in \mathbb{C}_{-}, \\
K(\lambda) & =\frac{D_{+}(\lambda)}{2 i \lambda} E(x,-\lambda)-\frac{D_{-}(\lambda)}{2 i \lambda} E(x, \lambda), \quad \lambda \in \mathbb{R}^{*},
\end{aligned}
$$

are the solutions of the boundary problem (1.5)-(1.6), where

$$
\hat{D}_{ \pm}(\lambda)=\left(A_{0}+A_{1} \lambda\right) \hat{E}_{x}^{ \pm}(0, \lambda)-\left(B_{0}+B_{1} \lambda\right) \hat{E}^{ \pm}(0, \lambda) .
$$

Now let us assume that

$$
Q \in A C\left(\mathbb{R}_{+}\right), \lim _{x \rightarrow \infty} Q(x)=0, \sup _{x \in[0, \infty)}\left[e^{\varepsilon \sqrt{x}}\left\|Q^{\prime}(x)\right\|\right]<\infty, \quad \varepsilon>0 .
$$

Theorem 1. Under the condition (3.10), the operator L has a finite number of eigenvalues and spectral singularities, and each of them is of finite multiplicity. 


\section{PRincipal FunCtions of $L$}

Under the condition (3.10), let $\lambda_{1}, \ldots, \lambda_{j}$ and $\lambda_{j+1}, \ldots, \lambda_{k}$ denote the zeros $H^{+}$ in $\mathbb{C}_{+}$and $H^{-}$in $\mathbb{C}_{-}$(which are the eigenvalues of $L$ ) with multiplicities $m_{1}, \ldots, m_{j}$ and $m_{j+1}, \ldots, m_{k}$, respectively. It is obvious that from the definition of the Wronskian

$$
\left\{\frac{d^{n}}{d \lambda^{n}} W\left[K^{+}(x, \lambda), E(x, \lambda)\right]\right\}_{\lambda=\lambda_{p}}=\left\{\frac{d^{n}}{d \lambda^{n}} D_{+}(\lambda)\right\}_{\lambda=\lambda_{p}}=0
$$

for $n=0,1, \ldots, m_{p}-1, p=1,2, \ldots, j$, and

$$
\left\{\frac{d^{n}}{d \lambda^{n}} W\left[K^{-}(x, \lambda), E(x,-\lambda)\right]\right\}_{\lambda=\lambda_{p}}=\left\{\frac{d^{n}}{d \lambda^{n}} D_{-}(\lambda)\right\}_{\lambda=\lambda_{p}}=0
$$

for $n=0,1, \ldots, m_{p}-1, p=j+1, \ldots, k$.

Theorem 2. The following formulae:

$$
\left\{\frac{\partial^{n}}{\partial \lambda^{n}} K^{+}(x, \lambda)\right\}_{\lambda=\lambda_{p}}=\sum_{m=0}^{n} F_{m}\left(\lambda_{p}\right)\left\{\frac{\partial^{m}}{\partial \lambda^{m}} E(x, \lambda)\right\}_{\lambda=\lambda_{p}},
$$

$n=0,1, \ldots, m_{p}-1, p=1,2, \ldots, j$, where

$$
\begin{gathered}
F_{m}\left(\lambda_{p}\right)=\left(\begin{array}{c}
n \\
m
\end{array}\right)\left\{\frac{\partial^{n-m}}{\partial \lambda^{n-m}} \hat{D}_{+}(\lambda)\right\}_{\lambda=\lambda_{p}}, \\
\left\{\frac{\partial^{n}}{\partial \lambda^{n}} K^{-}(x, \lambda)\right\}_{\lambda=\lambda_{p}}=\sum_{m=0}^{n} N_{m}\left(\lambda_{p}\right)\left\{\frac{\partial^{m}}{\partial \lambda^{m}} E(x,-\lambda)\right\}_{\lambda=\lambda_{p}},
\end{gathered}
$$

$n=0,1, \ldots, m_{p}-1, p=j+1, \ldots, k$, where

$$
N_{m}\left(\lambda_{p}\right)=\left(\begin{array}{c}
n \\
m
\end{array}\right)\left\{\frac{\partial^{n-m}}{\partial \lambda^{n-m}} \hat{D}_{-}(\lambda)\right\}_{\lambda=\lambda_{p}}
$$

hold.

Proof. We will prove only (4.3) using the method of induction, because the case of (4.5) is similar. Let be $n=0$. Since $K^{+}(x, \lambda)$ and $E(x, \lambda)$ are linearly dependent from (4.1), we get

$$
K^{+}\left(x, \lambda_{p}\right)=f_{0}\left(\lambda_{p}\right) E\left(x, \lambda_{p}\right),
$$

where $f_{0}\left(\lambda_{p}\right) \neq 0$. Let us assume that $1 \leq n_{0} \leq m_{p}-2$, (4.7) holds; that is,

$$
\left\{\frac{\partial^{n_{0}}}{\partial \lambda^{n_{0}}} K^{+}(x, \lambda)\right\}_{\lambda=\lambda_{p}}=\sum_{m=0}^{n_{0}} F_{m}\left(\lambda_{p}\right)\left\{\frac{\partial^{m}}{\partial \lambda^{m}} E(x, \lambda)\right\}_{\lambda=\lambda_{p}} .
$$


We will prove that (4.3) holds for $n_{0}+1$. If $Y(x, \lambda)$ is a solution of (1.5), then $\frac{\partial^{n}}{\partial \lambda^{n}} Y(x, \lambda)$ satisfies

$$
\left[-\frac{d^{2}}{d x^{2}}+Q(x)-\lambda^{2}\right] \frac{\partial^{n}}{\partial \lambda^{n}} Y(x, \lambda)=2 \lambda n \frac{\partial^{n-1}}{\partial \lambda^{n-1}} Y(x, \lambda)+n(n-1) \frac{\partial^{n-2}}{\partial \lambda^{n-2}} Y(x, \lambda) .
$$

Writing (4.9) for $K^{+}(x, \lambda)$ and $E(x, \lambda)$, and using (4.8), we find

$$
\left[-\frac{d^{2}}{d x^{2}}+Q(x)-\lambda^{2}\right] g_{n_{0}+1}\left(x, \lambda_{p}\right)=0,
$$

where

$$
g_{n_{0}+1}\left(x, \lambda_{p}\right)=\left\{\frac{\partial^{n_{0}+1}}{\partial \lambda^{n_{0}+1}} K^{+}(x, \lambda)\right\}_{\lambda=\lambda_{p}}-\sum_{m=0}^{n_{0}+1} F_{m}\left(\lambda_{p}\right)\left\{\frac{\partial^{m}}{\partial \lambda^{m}} E(x, \lambda)\right\}_{\lambda=\lambda_{p}} .
$$

From (4.1), we have

$$
W\left[g_{n_{0}+1}\left(x, \lambda_{p}\right), E\left(x, \lambda_{p}\right)\right]=\left\{\frac{d^{n_{0}+1}}{d \lambda^{n_{0}+1}} W\left[K^{+}(x, \lambda), E(x, \lambda)\right]\right\}_{\lambda=\lambda_{p}}=0 .
$$

Hence there exists a constant $f_{n_{0}+1}\left(\lambda_{p}\right)$ such that

$$
g_{n_{0}+1}\left(x, \lambda_{p}\right)=f_{n_{0}+1}\left(\lambda_{p}\right) E\left(x, \lambda_{p}\right) .
$$

This shows that (4.3) holds for $n=n_{0}+1$.

Using (4.3) and (4.5), define the functions

$$
U_{n, p}(x)=\left\{\frac{\partial^{n}}{\partial \lambda^{n}} K^{+}(x, \lambda)\right\}_{\lambda=\lambda_{p}}=\sum_{m=0}^{n} F_{m}\left(\lambda_{p}\right)\left\{\frac{\partial^{m}}{\partial \lambda^{m}} E(x, \lambda)\right\}_{\lambda=\lambda_{p}},
$$

$n=0,1, \ldots, m_{p}-1, p=1,2, \ldots, j$ and

$$
U_{n, p}(x)=\left\{\frac{\partial^{n}}{\partial \lambda^{n}} K^{-}(x, \lambda)\right\}_{\lambda=\lambda_{p}}=\sum_{m=0}^{n} N_{m}\left(\lambda_{p}\right)\left\{\frac{\partial^{m}}{\partial \lambda^{m}} E(x,-\lambda)\right\}_{\lambda=\lambda_{p}},
$$

$n=0,1, \ldots, m_{p}-1, p=j+1, \ldots, k$.

Then for $\lambda=\lambda_{p}, p=1,2, \ldots, j, j+1, \ldots, k$,

$$
\begin{aligned}
l\left(U_{0, p}\right) & =0, \\
l\left(U_{1, p}\right)+\frac{1}{1 !} \frac{\partial}{\partial \lambda} l\left(U_{0, p}\right) & =0, \\
l\left(U_{n, p}\right)+\frac{1}{1 !} \frac{\partial}{\partial \lambda} l\left(U_{n-1, p}\right)+\frac{1}{2 !} \frac{\partial^{2}}{\partial \lambda^{2}} l\left(U_{n-2, p}\right) & =0,
\end{aligned}
$$


$n=2,3, \ldots, m_{p}-1$,

hold, where $l(u)=-u^{\prime \prime}+Q(x) u-\lambda^{2} u$ and $\frac{\partial^{m}}{\partial \lambda^{m}} l(u)$ denote the differential expressions whose coefficients are the $m$-th derivatives with respect to $\lambda$ of the corresponding coefficients of the differential expression $l(u)$. (4.16) shows that $U_{0, p}$ is the eigenfunction corresponding to the eigenvalue $\lambda=\lambda_{p} ; U_{1, p}, U_{2, p}, \ldots U_{m_{p}-1, p}$ are the associated functions of $U_{0, p}[16,17]$.

$U_{0, p}, U_{1, p}, \ldots U_{m_{p}-1, p}, p=1,2, \ldots, j, j+1, \ldots, k$ are called the principal functions corresponding to the eigenvalue $\lambda=\lambda_{p}, p=1,2, \ldots, j, j+1, \ldots, k$ of $L$.

\section{Theorem 3.}

$$
U_{n, p} \in L_{2}\left(\mathbb{R}_{+}, E\right), \quad n=0,1, \ldots m_{p}-1, p=1,2, \ldots, j, j+1, \ldots, k .
$$

Proof. Let be $0 \leq n \leq m_{p}-1$ and $1 \leq p \leq j$. Using (2.5) and (3.10), we obtain that

$$
\|K(x, t)\| \leq c e^{-\sqrt{\frac{x+t}{2}}} .
$$

From (2.3), we get

$$
\left\|\left\{\frac{\partial^{n}}{\partial \lambda^{n}} E(x, \lambda)\right\}_{\lambda=\lambda_{p}}\right\| \leq x^{n} e^{-x \operatorname{Im} \lambda_{p}}+c \int_{x}^{\infty} t^{n} e^{-\sqrt{\frac{x+t}{2}}} e^{-t \operatorname{Im} \lambda_{p}} d t,
$$

where $c>0$ is a constant. Since $\operatorname{Im} \lambda_{p}>0$ for the eigenvalues $\lambda_{p}, p=1,2, \ldots, j$, of $L$, implies that

$$
\left\{\frac{\partial^{n}}{\partial \lambda^{n}} E(x, \lambda)\right\}_{\lambda=\lambda_{p}} \in L_{2}\left(\mathbb{R}_{+}, E\right), \quad n=0,1, \ldots m_{p}-1, p=1,2, \ldots, j .
$$

So we get $U_{n, p} \in L_{2}\left(\mathbb{R}_{+}, E\right)$ from (4.14) and (4.20) Similarly we prove the results for $0 \leq n \leq m_{p}-1, j+1 \leq p \leq k$. This completes the proof.

Let $\mu_{1}, \ldots, \mu_{v}$ and $\mu_{v+1}, \ldots, \mu_{l}$ be the zeros of $D_{+}$and $D_{-}$in $\mathbb{R}^{*}$ with multiplicities $n_{1}, \ldots, n_{v}$ and $n_{v+1}, \ldots, n_{l}$, respectively. We can show

$$
\left\{\frac{\partial^{n}}{\partial \lambda^{n}} K(x, \lambda)\right\}_{\lambda=\mu_{p}}=\sum_{m=0}^{n} C_{m}\left(\lambda_{p}\right)\left\{\frac{\partial^{m}}{\partial \lambda^{m}} E(x, \lambda)\right\}_{\lambda=\mu_{p}},
$$

$n=0,1, \ldots, n_{p}-1, p=1,2, \ldots, v$, where

$$
\begin{gathered}
C_{m}\left(\mu_{p}\right)=-\left(\begin{array}{c}
n \\
m
\end{array}\right)\left\{\frac{\partial^{n-m}}{\partial \lambda^{n-m}} D_{-}(\lambda)\right\}_{\lambda=\mu_{p}}, \\
\left\{\frac{\partial^{n}}{\partial \lambda^{n}} K(x, \lambda)\right\}_{\lambda=\mu_{p}}=\sum_{m=0}^{n} R_{m}\left(\mu_{p}\right)\left\{\frac{\partial^{m}}{\partial \lambda^{m}} E(x,-\lambda)\right\}_{\lambda=\mu_{p}},
\end{gathered}
$$


$n=0,1, \ldots, n_{p}-1, p=v+1, \ldots, l$,

where

$$
R_{m}\left(\mu_{p}\right)=\left(\begin{array}{c}
n \\
m
\end{array}\right)\left\{\frac{\partial^{n-m}}{\partial \lambda^{n-m}} D_{+}(\lambda)\right\}_{\lambda=\mu_{p}} .
$$

Now define the generalized eigenfunctions and generalized associated functions corresponding to the spectral singularities of $L$ by the following :

$$
V_{n, p}(x)=\left\{\frac{\partial^{n}}{\partial \lambda^{n}} K(x, \lambda)\right\}_{\lambda=\mu_{p}}=\sum_{m=0}^{n} C_{m}\left(\mu_{p}\right)\left\{\frac{\partial^{m}}{\partial \lambda^{m}} E(x, \lambda)\right\}_{\lambda=\mu_{p}},
$$

$n=0,1, \ldots, n_{p}-1, p=1,2, \ldots, v$,

$$
V_{n, p}(x)=\left\{\frac{\partial^{n}}{\partial \lambda^{n}} K(x, \lambda)\right\}_{\lambda=\mu_{p}}=\sum_{m=0}^{n} R_{m}\left(\mu_{p}\right)\left\{\frac{\partial^{m}}{\partial \lambda^{m}} E(x,-\lambda)\right\}_{\lambda=\mu_{p}},
$$

$n=0,1, \ldots, n_{p}-1, p=v+1, \ldots, l$.

Then $V_{n, p}, n=0,1, \ldots, n_{p}-1, p=1,2, \ldots, v, v+1, \ldots, l$, also satisfy the equations analogous to (4.16).

$V_{0, p}, V_{1, p}, \ldots, V_{n_{p}-1, p}, p=1,2, \ldots, v, v+1, \ldots, l$ are called the principal functions corresponding to the spectral singularities $\lambda=\mu_{p}, p=1,2, \ldots, v, v+1, \ldots, l$ of $L$.

\section{Theorem 4.}

$$
V_{n, p} \notin L_{2}\left(\mathbb{R}_{+}, E\right), \quad n=0,1, \ldots n_{p}-1, p=1,2, \ldots, v, v+1, \ldots, l .
$$

Proof. For $0 \leq n \leq n_{p}-1$ and $1 \leq p \leq v$ using (2.3), we obtain

$$
\left\|\left\{\frac{\partial^{n}}{\partial \lambda^{n}} E(x, \lambda)\right\}_{\lambda=\mu_{p}}\right\| \leq\left\|(i x)^{n} e^{i \mu_{p} x} I+\int_{x}^{\infty}(i t)^{n} K(x, t) e^{i \mu_{p} t} d t\right\|
$$

since $\operatorname{Im} \mu_{p}=0, p=1,2, \ldots, v$, we find that

$$
\int_{0}^{\infty}\left\|(i x)^{n} e^{i \mu_{p} x} I\right\|^{2} d x=\int_{0}^{\infty} x^{2 n}=\infty .
$$

So we obtain $V_{n, p} \notin L_{2}\left(\mathbb{R}_{+}, E\right), n=0,1, \ldots n_{p}-1, p=1,2, \ldots, v$. Using the similar way, we may also prove the results for $0 \leq n \leq n_{p}-1, v+1 \leq p \leq l$.

Now define the Hilbert spaces of vector-valued functions with values in $E$ by

$$
H_{n}:=\left\{f: \int_{0}^{\infty}(1+|x|)^{2 n}\|f(x)\|^{2} d x<\infty\right\}, n=1,2, \ldots,
$$




$$
H_{-n}:=\left\{g: \int_{0}^{\infty}(1+|x|)^{-2 n}\|g(x)\|^{2} d x<\infty\right\}, n=1,2, \ldots,
$$

with the norms

$$
\|f\|_{n}^{2}:=\int_{0}^{\infty}(1+|x|)^{2 n}\|f(x)\|^{2} d x
$$

and

$$
\|g\|_{-n}^{2}:=\int_{0}^{\infty}(1+|x|)^{-2 n}\|g(x)\|^{2} d x,
$$

respectively. Then

$$
H_{n+1} \varsubsetneqq H_{n} \varsubsetneqq L_{2}\left(\mathbb{R}_{+}, E\right) \varsubsetneqq H_{-n} \varsubsetneqq H_{-(n+1)}, \quad n=1,2, \ldots,
$$

and $H_{-n}$ is isomorphic to the dual of $H_{n}$.

\section{Theorem 5.}

$$
V_{n, p} \in H_{-(n+1)}, \quad n=0,1, \ldots n_{p}-1, p=1,2, \ldots, v, v+1, \ldots, l .
$$

Proof. For $0 \leq n \leq n_{p}-1$ and $1 \leq p \leq v$ using (2.3) and (4.24), we get

$$
\begin{aligned}
& \int_{0}^{\infty}(1+|x|)^{-2(n+1)}\left\|V_{n, p}\right\|^{2} d x \\
& \leq M \int_{0}^{\infty}(1+|x|)^{-2(n+1)}\left\{\left\|\{E(x, \lambda)\}_{\lambda=\mu_{p}}\right\|^{2}+\ldots+\left\|\left\{\frac{\partial^{n}}{\partial \lambda^{n}} E(x, \lambda)\right\}_{\lambda=\mu_{p}}\right\|^{2}\right\}
\end{aligned}
$$

where $M>0$ is a constant. Using (2.3), we have

$$
\int_{0}^{\infty}(1+|x|)^{-2(n+1)}\left\|(i x)^{n} e^{i \mu_{p} x} I\right\|^{2} d x<\infty
$$

and

$$
\int_{0}^{\infty}(1+|x|)^{-2(n+1)}\left\|\int_{x}^{\infty}(i t)^{n} K(x, t) e^{i \mu_{p} t} d t\right\|^{2} d x<\infty .
$$

Consequently $V_{n, p} \in H_{-(n+1)}$ for $0 \leq n \leq n_{p}-1$ and $1 \leq p \leq v$. Similarly, we obtain $V_{n, p} \in H_{-(n+1)}$ for $0 \leq n \leq n_{p}-1$ and $v+1 \leq p \leq l$.

Let us choose

$$
n_{0}=\max \left\{n_{1}, n_{2}, \ldots, n_{v}, n_{v+1}, \ldots, n_{l}\right\} .
$$

By (4.28), we get the following 


\section{Theorem 6.}

$$
V_{n, p} \in H_{-n_{0}}, \quad n=0,1, \ldots n_{p}-1, p=1,2, \ldots, v, v+1, \ldots, l .
$$

\section{REFERENCES}

[1] Z. S. Agranovich and V. A. Marchenko, The Inverse Problem of Scattering Theory, 2nd ed., ser. Series is books. London: Gordon and Breach, 1963.

[2] Y. Aygar and E. Bairamov, "Jost solution and the spectral properties of the matrix-valued difference operators," Appl. Math. and Comput., vol. 218, no. 3, pp. 9676-9681, 2012.

[3] E. Bairamov, Y. Aygar, and T. Koprubasi, "Spectrum of the eigenparameter dependent discrete sturm-liouville equations," Czechoslovak Math. J., vol. 49, pp. 689-700, 1999.

[4] E. Bairamov, O. Cakar, and A. O. Celebi, "Quadratic pencil of schröndinger operators with spectral singularities: discrete spectrum and principal functions," J. Math. Anal. Appl., vol. 216, pp. 303-320, 1997.

[5] E. Bairamov, O. Cakar, and A. M. Krall, "An eigenfunction expansion for a quadratic pencil of a scrödinger operator with spectral singularities," J. Differential Equations, vol. 151, pp. 268-289, 1999.

[6] E. Bairamov, O. Cakar, and A. M. Krall, "Spectrum and spectral singularities of a quadratic pencil of a schrödinger operator with a general boundary condition," J. Differential Equations, vol. 151 no.2, pp. 252-267, 1999.

[7] E. Bairamov and A. O. Çelebi, "Spectral properties of the klein-gordon s-wave equation with complex potential," Indian J. Pure Appl. Math., vol. 28, pp. 813-824, 1997.

[8] E. Bairamov and A. O. Celebi, "Spectrum and spectral expansion for the non-selfadjoint discrete dirac operators," Quart. J. Math. Oxford Ser., vol. (2) no. 200, pp. 371-384, 1999.

[9] E. Bairamov and S. Seyyidoğlu, "Non-selfadjoint singular sturm-liouville problems with boundary conditions dependent on the eigenparameter," Abstr. Appl. Anal.,, vol. 2010, pp. 1-10, 2010.

[10] E. Bairamov and G. B. Tunca, "Discrete spectrum and principal functions of non-selfadjoint differential operators," Journal of Computational and Applied Mathematics, vol. 235, pp. 4519-4523, 2011.

[11] R. Carlson, "An inverse problem for the matrix schrödinger equation," J. Math. Anal. Appl., vol. 267, pp. 564-575, 2002

[12] S. Clark and F. Gesztesy, "Weyl-titchmarsh m-function asymptotics, local uniqueness results, trace formulas and borg-type theorems for dirac operators," Trans Amer. Math. Soc., vol. 354, pp. 34753534, 2002.

[13] S. Clark, F. Gesztesy, and W. Renger, "Trace formulas and borg-type theorems for matrix-valued jacobi and dirac finite difference operators," J. Differential Equations, vol. 219, pp. 144-182, 2005.

[14] C. Coskun and M. Olgun, "Principal functions of non-selfadjoint matrix sturm-liouville equations," Journal of Computational and Applied Mathematics, vol. 235 no. 16, pp. 4834-4838, 2011.

[15] F. Gesztesy, A. Kiselev, and K. A. Makarov, "Uniqueness results for matrix-valued schrödinger, jacobi and dirac-type operators," Math. Nachr., vol. 239, pp. 103-145, 2002.

[16] M. V. Keldysh, "On the completeness of the eigenfunctions of some classes of non-selfadjoint linear operators," Soviet Mathematics-Doklady, vol. 77 no. 4, pp. 11-14, 1951.

[17] M. V. Keldysh, "On the completeness of the eigenfunctions of some classes of non-selfadjoint linear operators," Russian Mathematical Surveys, vol. 26 no. 4, pp. 15-41, 1971.

[18] A. M. Krall, E. Bairamov, and O. Cakar, "Spectral analysis of a non-selfadjoint discrete schrödinger operators with spectral singularities," Math. Nachr., vol. 231, pp. 89-104, 2001.

[19] V. E. Lyance, "A differential operator with spectral singularities, i, ii,," AMS Translations, vol. 60, pp. 185-225, 227-283, 1967. 
[20] M. A. Naimark, "Investigation of the spectrum and the expansion in eigenfunctions of a nonselfadjoint operators of second order on a semi-axis," AMS Translations, vol. 16, pp. 103-193, 1960.

[21] M. Olgun and C. Coskun, "Non-selfadjoint matrix sturm-liouville operators with spectral singularities," Applied Mathematics and Computations, vol. 216 no.8, pp. 2271-2275, 2010.

Authors' addresses

Deniz Katar

Ankara University, Faculty of Sciences, Department of Mathematics, Ankara, Turkey

E-mail address: deniz.ktr@hotmail.com

\section{Murat Olgun}

Ankara University, Faculty of Sciences, Department of Mathematics, Ankara, Turkey

E-mail address: olguneankara.edu.tr

\section{Cafer Coskun}

Ankara University, Faculty of Sciences, Department of Mathematics, Ankara, Turkey

E-mail address: ccoskun@ankara.edu.tr 